\title{
Efficiency of Class I and Class II malocclusion treatment with four premolar extractions
}

\author{
Guilherme JANSON, Alexandre NAKAMURA, Sérgio Estelita BARROS, Roberto BOMBONATTI, Kelly CHIQUETO
}

Department of Pediatric Dentistry, Orthodontics and Community Health, Bauru School of Dentistry, University of São Paulo, Bauru, SP, Brazil.

Corresponding address: Dr. Guilherme Janson - Departamento de Odontopediatria, Ortodontia e Saúde Coletiva - Faculdade de Odontologia de Bauru - Universidade de São Paulo - Alameda Octávio Pinheiro Brisolla 9-75 - Bauru - SP - 17012-901 - Brazil - Phone/Fax: 551432344480 - e-mail: jansong@ travelnet.com.br

Submitted: September 26, 2013 - Modification: December 25, 2013 - Accepted: March 17, 2014

\section{ABSTRACT}

\begin{abstract}
Eour premolar extractions is a successful protocol to treat Class I malocclusion, but it is a less efficient way when compared with other Class II treatment protocols. Objective: The objective of this study was to evaluate the influence of anteroposterior discrepancy on the success of four premolar extractions protocol. For that, treatment efficiencies of Class I and complete Class II malocclusions, treated with four premolar extractions were compared. Methods: A sample of 107 records from 75 Class I (mean age of 13.98 years - group 1 ) and 32 Class II (mean age of 13.19 years - group 2) malocclusion patients treated with four premolar extractions was selected. The initial and final occlusal status of each patient was evaluated on dental casts with the PAR index. The treatment time was calculated based on the clinical charts, and the treatment efficiency was obtained by the ratio between the percentage of PAR reduction and treatment time. The PAR index and its components, the treatment time and the treatment efficiency of the groups were statistically compared with t tests and Mann-Whitney U-test. Results: The Class II malocclusion patients had a greater final PAR index than Class I malocclusion patients, and similar duration (Class I - 28.95 months. and Class II - 28.10 months.) and treatment efficiency. Conclusion: The treatment of the complete Class II malocclusion with four premolar extractions presented worse occlusal results than Class I malocclusion owing to incomplete molar relationship correction.
\end{abstract}

Keywords: Treatment protocols. Efficiency. Tooth extraction.

\section{NTRODUCTI ON}

Class II and Class III malocclusions have in common a molar relationship discrepancy, which can or cannot require correction depending on the treatment protocol ${ }^{8,13,26}$. When treatment is planned with or without extraction of four premolars, a Class I molar relationships is expected at the end of treatment. However, if only two premolars are extracted in a single arch, the initial molar relationship discrepancy should be maintained, while the other occlusal characteristics are normalized ${ }^{4,8,14,16,26}$.

Efficiency of Class II malocclusion correction has been compared between the different treatment protocols for this malocclusion ${ }^{13,21,22,28}$. The two premolar extractions protocol presented better occlusal results in a shorter treatment time when compared with non-extraction and four premolar extractions protocols, probably due to difficulties associated with molar relationship correction in these treatment approaches ${ }^{13,14,18}$. However, this speculation cannot be easily demonstrated because the comparisons were performed between different treatment protocols.

Thus, this study was designed to compare cases with and without requirement of molar relationship correction, but using the same treatment protocol. For that, treatment efficiency of Class I and complete Class II malocclusions, treated with four premolar extractions were compared.

\section{MATERIAL AND METHODS}

The sample size was calculated considering a value of $5 \%$ and $20 \%$ for a (type I error) and $\beta$ (type II error). The value of the measurement variance $\sigma 2$ (standard deviation) was based on a previous study ${ }^{7}$. Since weighted PAR score ranging of 2 has clinically significant impact on occlusal 
relationship, it was the minimum difference to be detected between groups ${ }^{29}$. Thus, a minimum of 16 patients in each group was required. The sample was retrospectively selected from the files of the Orthodontic Department at Bauru School of Dentistry. According to the objectives of this study, the primary inclusion criteria was patient records presenting Class I and complete bilateral Class II division 1 malocclusions treated with four first premolar extractions and standard fixed edgewise appliances. Additionally, the patients should present all permanent teeth up to the first molars and no dental anomalies of number, size, and form. Patients had not been submitted to RME or orthodontic-surgical approach. Considering these criteria, 75 Class I (47 female, 28 male - group 1) and 32 Class II (14 female, 18 male - group 2) malocclusion patients were selected from all treated and documented patients. Group 1 presented an initial mean age of $13.98 \pm 2.08$ years (range, 10.54-23.13 years) and group 2 had an initial mean age of $13.19 \pm 1.58$ years (range, 10.48-18.58 years). Considering that patients had unequal distribution between the groups ( $1: 2)$, the sample size was recalculated to compensate this specific disproportion. A total sample size with a minimum of 48 patients was required $(\mathrm{N}=16$ and $\mathrm{N}=32$ ), confirming that the number of selected patients was adequate.

Orthodontic mechanics included $0.022 \times 0.028$ inch fixed standard edgewise appliances, and extraction of four first premolars. Cases with severe initial anterior tooth crowding required initial canine retraction, followed by leveling and alignment with the usual wire sequence characterized by an initial 0.015 -inch twist-flex or a 0.016 -inch nitinol, followed by $0.016,0.018$, 0.020 , and $0.019 \times 0.025$-inch stainless steel archwire (Unitek, Monrovia, California, USA). Deep bite was corrected with accentuated and reversed curve of Spee. The extraction spaces were closed with "en masse" retraction of the anterior teeth, with elastic chains on a rectangular archwire. Class II patients used extraoral appliance for anchorage reinforcement and anteroposterior relationship correction. When necessary, Class II elastics were also used to aid in Class II anteroposterior correction.

The patients' records were used to determine their initial age (I-Age), sex, date of treatment onset, date of treatment completion, and total treatment time (TT). The initial and final occlusal statuses were evaluated using the peer assessment rating (PAR) index ${ }^{29}$, which was calculated on the pretreatment and posttreatment dental study models of each patient, according to the American weightings suggested by DeGuzman, et al. ${ }^{6}$ (1995). Initial and final occlusal characteristics were ranked by scores for molar and premolar AP relationship, overjet, overbite, midline, and crowding to quantify the initial malocclusion severity (I-PAR), the occlusal treatment results (F-PAR), and the percentage of PAR reduction (PCPAR), which is a better estimate of occlusal improvement ${ }^{11}$.

Since the PAR index analyzes a set of occlusal characteristics at the same time and does not discriminate the degree of participation of each in the total score, the posttreatment scores obtained for each PAR component were compared individually to determine the success rate achieved. Therefore, the PAR score at the end of treatment was separated into its several components to allow an individual evaluation. The treatment efficiency index (TEI) was evaluated by the relationship between PCPAR and TT in months, expressed as TEI $=$ PCPAR/TT 13 . The TEI increased when a greater PCPAR and/or a shorter $\Pi \mathrm{T}$ was observed.

\section{Error study}

Initial and final PAR scores were recalculated by the same examiner (AYN) in the pretreatment and posttreatment study models of 20 randomly selected patients after 30 days from the first measurement. The casual errors were estimated by Dahlberg's formula $\left(S e^{2}=\Sigma d^{2} / 2 n\right.$ ), where $S^{2}$ is the error variance and $d$ is the difference between 2 determinations of the same variable; the systematic errors were calculated with dependent t tests, at $\mathrm{P}<0.05$.

Table 1- Compatibility of Class I and complete Class II malocclusion groups regarding sex and age

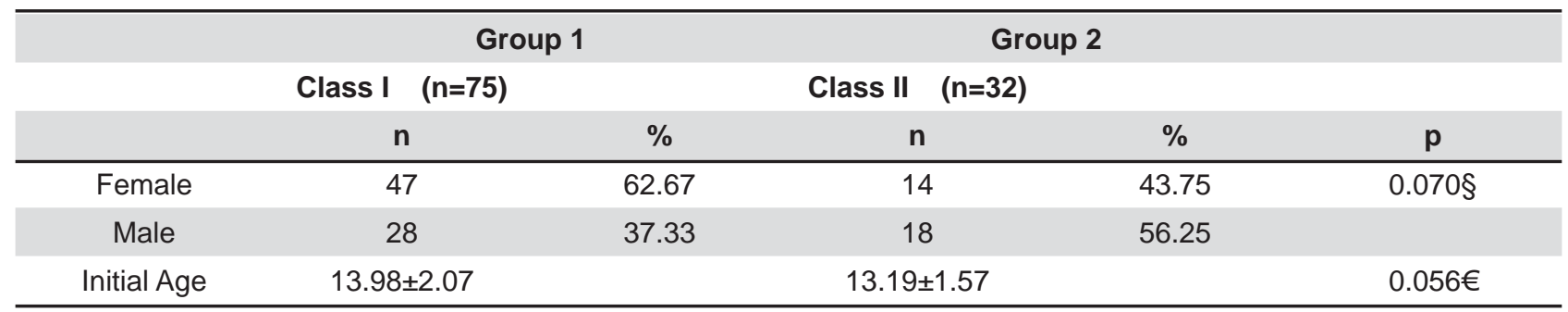

$\S$ Chi-square test

$€ \mathrm{t}$ test 


\section{Statistical analyses}

Compatibility of the groups regarding the proportion of sexes was evaluated with chi-square tests, while the I-Age similarity between the groups was evaluated with t test.

The PAR index variables (I-PAR; F-PAR; PCPAR) were compared between the groups using t tests.

The occlusal results obtained for each component of the PAR index were individually compared between the groups with the MannWhitney U-test. A nonparametric test was used because the values of each PAR component did not show normal distribution. The percentage of contribution from each PAR component to the total value of the F-PAR was also calculated.

The treatment time and treatment efficiency index were compared with t tests, and the influence of the variables Molar Relationship (MR); Sex (S); I-Age; I-PAR, F-PAR and PcPAR on treatment time was evaluated by multiple linear regression analysis.

\section{RESULTS}

The PAR evaluation did not present a significant systematic error, and the casual error was within acceptable level $(P A R=1.5)$. The groups were similar regarding the proportion of sexes, initial age and treatment time (Table 1 ).

The initial severity (I-PAR) of Class I and Class II malocclusions were similar in the groups. The better occlusal results and success rate were achieved in group 1 , that had a significantly smaller F-PAR and a greater PCPAR (Table 2).

Molar and premolar AP relationship was the only final PAR index component that showed statistically significant difference between the groups, and its greater score value represents a worse AP relationship obtained in group 2. Molar and premolar AP relationship contributed only with $23 \%$ of the total F-PAR value obtained in Group 1 , while $43.95 \%$ of the total F-PAR value obtained in Group 2 was due to it (Table 3).

Treatment time (TT) and treatment efficiency index (TEI) were similar in the groups. The variables that comprised the regression analysis model showed no significant influence on treatment time, and they had a low predictive value to explain the duration of orthodontic treatment (Table 4).

Table 2 - Comparison of Class I and complete Class II malocclusion groups regarding PAR score

\begin{tabular}{|c|c|c|c|c|c|}
\hline & \multicolumn{2}{|c|}{ Class I $\quad(n=75)$} & \multicolumn{2}{|c|}{ Class II $\quad(n=32)$} & \multirow[b]{2}{*}{$\mathbf{P}$} \\
\hline & Mean & SD & Mean & SD & \\
\hline I-PAR & 23.64 & 5.45 & 26.18 & 7.53 & 0.052 \\
\hline F-PAR & 2.40 & 3.08 & 5.12 & 5.69 & $0.001^{*}$ \\
\hline PcPAR & 89.46 & 14.39 & 79.18 & 24.58 & $0.008^{*}$ \\
\hline
\end{tabular}

* Statistically significant at $\mathrm{P}<0.05$.

I-PAR=initial malocclusion severity

F-PAR $=$ occlusal treatment results

PcPAR=percentage of PAR reduction

Table 3- Comparison of Class I and complete Class II malocclusion groups regarding F-PAR individual components, and proportion of each PAR component in F-PAR

\begin{tabular}{|c|c|c|c|c|c|c|}
\hline & Class I & $(n=75)$ & Class II & $(n=32)$ & & \\
\hline & $\%$ & Mean Rank & $\%$ & Mean Rank & $\mathbf{Z}$ & (U) \\
\hline AP & 23.33 & 50.05 & 43.95 & 63.32 & -2.755 & $0.005^{*}$ \\
\hline Overjet & 30.42 & 52.54 & 33.40 & 57.42 & -1.174 & 0.240 \\
\hline Overbite & 28.33 & 52.51 & 20.12 & 57.46 & -1.006 & 0.314 \\
\hline Midline & 3.33 & 54.42 & 0 & 53.00 & 0.928 & 0.353 \\
\hline Crowding & 14.17 & 56.21 & 2.34 & 48.81 & 1.578 & 0.114 \\
\hline
\end{tabular}

U Mann-Whitney U test

* Statistically significant at $\mathrm{P}<0.05$.

F-PAR=occlusal treatment results 
Table 4- Comparison of Class I and complete Class II malocclusion groups regarding treatment time (TT - in months) and treatment efficiency index (TEI), and multiple regression analysis with treatment time as the dependent variable

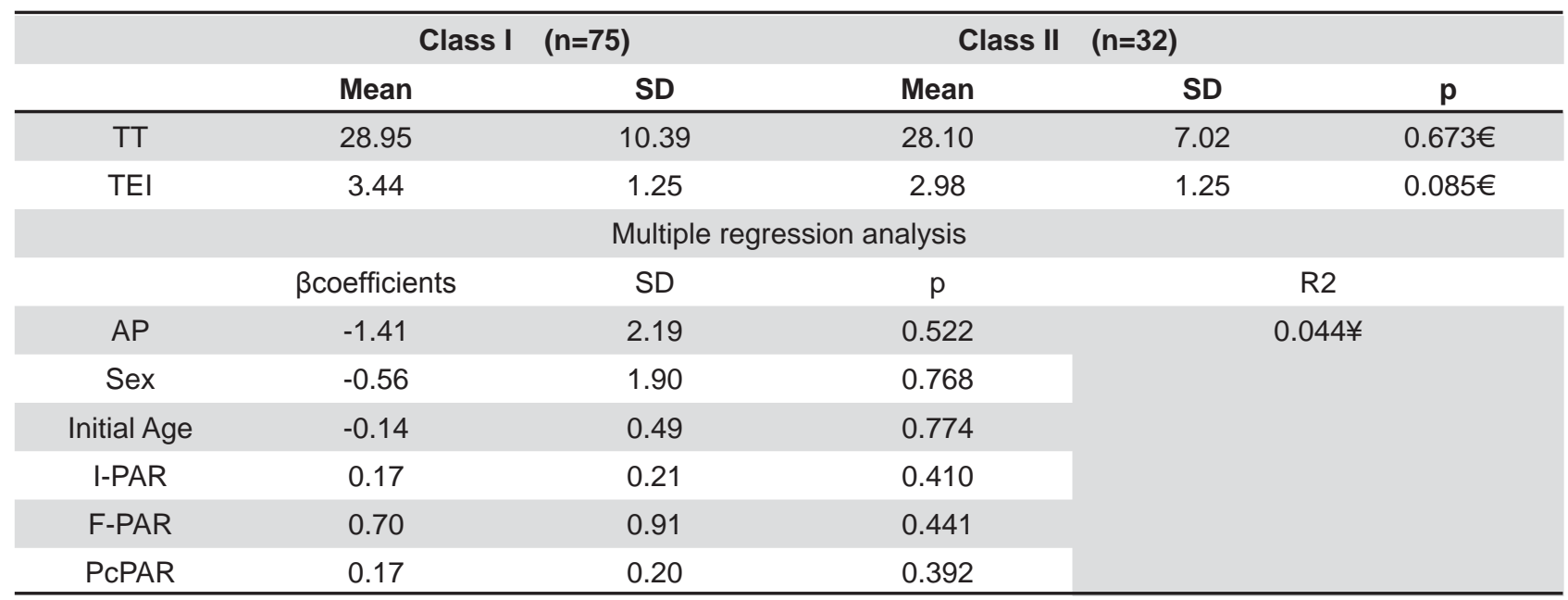

$€ \mathrm{t}$ test

$¥$ Multiple regression analysis

I-PAR=initial malocclusion severity

F-PAR=occlusal treatment results

PcPAR=percentage of PAR reduction

\section{DISCUSSION}

Molar relationship correction is an essential objective of Class II malocclusion treatment with four premolar extractions, which can be influenced by some factors. Patient sex and age is associated with the craniofacial growth potential, and patient compliance degree can also change according to these variables $9,20,30$. Considering that patient growth and compliance influence Class II malocclusion correction, compatibility of the groups regarding sexes proportion and initial age allowed an unbiased molar relationship correction evaluation (Table 1). Although not significantly, the slightly smaller initial mean age and the greater male patient proportion of group 2 benefited Class II malocclusion treatment since, at this mean age range, Class II treatment at a younger age is more favorable because there will be greater growth changes that can be redirected with treatment ${ }^{10,23}$. Males are benefited in Class II treatment at this mean age because their growth spurt occurs later than in females, and occurs a little later than the initial mean age reported. Therefore, treatment was conducted in a large number of males during their peak growth spurt ${ }^{10,23}$. However, this was not enough to ensure a similar occlusal result to group 1.

The similar initial malocclusion severity observed in the groups could be considered an unexpected result because Class II malocclusion has an anteroposterior discrepancy that is not present in Class I (Table 2). However, the overjet was the only different malocclusion trait between the groups. Therefore, it was not enough to produce a significant intergroup discrepancy regarding the total PAR value.

The worst occlusal result and PCPAR correction of Class II malocclusion treatment does not seem to be associated with the extraction protocol because both were treated with fourpremolar extractions (Table 2). Incisor crowding or labial tipping is easily solved during anterior retraction and they would unlikely affect the PCPAR correction. However, Class II treatment with or without four premolar extractions always require molar relationship correction, and a deficient anteroposterior correction can affect the PCPAR correction 4,12-14,17,19. Thus, if initially there is a Class I relationship, as in group 1, four premolar extractions will not affect the treatment results, but if there is a complete initial Class II molar relationship, as in group 2, its incomplete correction can compromise the treatment results.

This inherent difficulty of molar relationship correction significantly influenced the treatment results and the PCPAR correction of the Class II group. The AP relationship was the only F-PAR component that was significantly worse for the Class II group at the end of treatment (Table 3). The AP relationship of Class I and complete Class II malocclusions are equally scored with the PAR index because Class II molar relationship is considered an adequate final occlusion when only maxillary premolars are extracted, with successfully corrected canine relationship, overjet and overbite ${ }^{26}$. Thus, the initial AP relationship 
score was similar between the groups, but at the end of orthodontic treatment the AP relationship was deficient in group 2 due to the difficulty to treat a complete Class II to a Class I relationship ${ }^{13,14}$. In fact, the final AP relationship score represented almost half of the F-PAR in group 2, while group 1 showed an F-PAR value with a more equilibrated score distribution among the PAR components, without a predominant factor determining treatment limitations (Table 3 ).

Although the PCPAR reduction was smaller in the Class II than in the Class I group due to the difficulty to correct molar relationship, treatment time was similar in the groups, probably because orthodontic treatment was finished before total Class II correction (Table 4). This similar treatment time contributed to a similar treatment efficiency index (TEI) in the groups in spite of the smaller PcPAR reduction of the Class II group. Perhaps, if group 2 patients were treated up to a better molar relationship, its treatment time would be longer than the Class I group. However, when the occlusal results are essentially dependent on patient compliance, a longer treatment time does not always mean an actual treatment progress towards planned objectives if the patient is not engaged or concerned in obtaining the best results that orthodontic treatment can provide. Consequently, and contrary to common sense, longer treatments are frequently associated with less satisfactory results, and additional active treatment could not improve the results of noncompliant patients ${ }^{27}$. In these cases, two options should be considered by the professional in the patient's best interest: 1- orthodontic treatment of noncompliant patients should not be continued in the hope of attaining a better result 27 ; 2- whenever possible, a compliance free appliance could be attempted ${ }^{24}$, although minimal patient cooperation and caution with hygiene, appointments and appliance breakage will always be necessary to adequately conduct orthodontic treatment ${ }^{27}$.

The regression analysis showed that none of the independent variables explained treatment time satisfactorily (Table 4). This absence of correlation between treatment time and success rate corroborates the thought that treatment protocols with high requirement of patient compliance have low success predictability regardless of the treatment time spent $5,13,14,24,27$. Thus, molar relationship correction did not contribute significantly to explain treatment duration because some patients had, simultaneously, longer treatment time and greater F-PAR score due to unsatisfactory correction of molar relationship. AP correction was not improved when treatment time was extended in the effort of obtaining a better treatment result. Briefly, if patient compliance is not obtained, treatment time will have no influence on AP correction when Class II mechanics is fully patient-dependent. Other variables had yet smaller predictive values to determine treatment time because, theoretically, obtaining an ideal occlusion is usually taken as a strong clinical parameter to determine appliance removal. But, if an ideal occlusion cannot be obtained, treatment ending will become a subjective decision and treatment time can vary according to unconventional parameters.

\section{Clinical implications}

Some occlusal objectives of orthodontic treatment are inherent to the protocol choice. Thus, if four premolar extractions protocol is chosen, a Class I molar relationship should be established at the end of treatment regardless of the initial anteroposterior relationship $p^{4,25,26}$. Because of the demonstrated difficulty to correct the AP relationship, a four premolar extractions protocol should be used with caution when the initial molar relationship is severely displaced from Class I and patient growth potential is reduced or absent. In these cases, premolar extractions in a single arch can be the best choice concerning occlusion. This extraction protocol does not require molar relationship changes to correct canine relationship and overjet, increasing the predictability of the occlusal results due to the smaller need of patient compliance with anchorage reinforcement and intermaxillary elastics 3,4,14,15.

In Class II malocclusions, even when the orthodontist is convinced that patient compliance will be good and that the growth potential could help to achieve a Class I molar relationship, the four premolar extractions protocol should be used with caution, since it produces greater incisor retraction than two maxillary premolar extractions ${ }^{1,4,14}$, and the patient profile may not be benefited with these soft tissue changes. Thus, orthodontists should definitely include the two premolar extractions protocol in their treatment options. However, it is necessary to have in mind that this protocol requires specific orthodontic mechanic guidance on how to correctly position the teeth when a final Class II molar relationship is intended, allowing the establishment of an excellent static and functional occlusion with smaller incisor retraction, soft tissue changes, patient compliance needs and unsuccessful results $2-4,14-16,26,31$.

\section{CONCLUSIONS}

Class I malocclusions treated with four premolar extractions had better occlusal treatment results 
and greater success rate than complete Class II malocclusions similarly treated;

Molar relationship correction was the unsuccessful treatment objective that primarily contributed for a more deficient occlusal result in complete Class II malocclusions;

Treatment time similarity was the determinant factor for the similar treatment efficiency indexes of both malocclusions.

\section{REFERENCES}

1- Andrews LF. The straight wire appliance. Syllabus of philosophy and techniques. techniques. $2^{\text {nd }}$ ed. San Diego: L. F. Andrews; 1975. p. 109-41.

2- Bokas J, Collett T. Effect of upper premolar extractions on the position of the upper lip. Aust Orthod J. 2006; 22:31-7.

3- Booij JW, Kuijpers-J agtman AM, Katsaros C. A treatment method for Class II Division 1 patients with extraction of permanent maxillary first molars. World J Orthod. 2009;10:41-8.

4- Bryk C, White LW. The geometry of Class II correction with extractions. J Clin Orthod. 2001;35:570-9.

5- Casutt C, Pancherz H, Gawora M, Ruf S. Success rate and efficiency of activator treatment. Eur J Orthod. 2007;29:614-21. 6- DeGuzman L, Bahiraei D, Vig KW, Vig PS, Weyant RJ, O'Brien $K$. The validation of the Peer Assessment Rating index for malocclusion severity and treatment difficulty. Am J Orthod Dentofac Orthop. 1995; 107: 172-6.

7- Dyken RA, Sadowsky PL, Hurst D. Orthodontic outcomes assessment using the peer assessment rating index. Angle Orthod. 2001; 71: 164-9.

8- Farret MM, Farret MM, Farret AM. Strategies to finish orthodontic treatment with a Class III molar relationship: three patient reports. World J Orthod. 2009; 10:323-33.

9- Hägg $U$, Taranger J. Maturation indicators and the pubertal growth spurt. Am J Orthod. 1982;82:299-309.

10- Harris EF. Effects of patient age and sex on treatment: correction of Class II malocclusion with the Begg technique. Angle Orthod. 2001; 71:433-41.

11- Holman JK, Hans MG, Nelson S, Powers MP. An assessment of extraction versus nonextraction orthodontic treatment using the peer assessment rating (PAR) index. Angle Orthod. 1998; 68:52734.

12- Jacobs T, Sawaengkit P. National Institute of Dental and Craniofacial Research efficacy trials of bionator Class II treatment: a review. Angle Orthod. 2002; 72:571-5.

13- Janson G, Barros SE, Freitas MR, Henriques JF, Pinzan A. Class II treatment efficiency in maxillary premolar extraction and nonextraction protocols. Am J Orthod Dentofacial Orthop. 2007; 132:490-8.

14- Janson G, Brambilla AC, Henriques JF, Freitas MR, Neves LS. Class II treatment success rate in 2- and 4-premolar extraction protocols. Am J Orthod Dentofac Orthop. 2004; 125:472-9.

15- Janson G, Dainesi EA, Henriques JF, Freitas MR, Lima KJ. Class II subdivision treatment success rate with symmetric and asymmetric extraction protocols. Am J Orthod Dentofac Orthop. 2003; 124: 257-64.
16- Janson G, Fuziy A, Freitas MR, Castanha Henriques JF, Almeida RR. Soft-tissue treatment changes in Class II Division 1 malocclusion with and without extraction of maxillary premolars. Am J Orthod Dentofacial Orthop. 2007; 132: 729 e1-8.

17- Janson G, Graciano JT, Henriques JF, Freitas MR, Pinzan A, Pinzan-Vercelino CR. Occlusal and cephalometric Class II Division 1 malocclusion severity in patients treated with and without extraction of 2 maxillary premolars. Am J Orthod Dentofacial Orthop. 2006; 129: 759-67.

18- Janson G, Maria FRT, Barros SEC, Freitas M, Henriques J FC. Orthodontic treatment time in 2- and 4-premolar-extraction protocols. Amer J Orthod Dentofac Ortop. 2006; 129:666-771.

19- Janson G, Valarelli FP, Cançado RH, Freitas MR, Pinzan A. Relationship between malocclusion severity and treatment success rate in Class II nonextraction therapy. Am J Orthod Dentofacial Orthop. 2009; 135: 274 e1-8; discussion 274-5.

20- John W, Kerr S, Buchanan IB, McNair FI, McColl JH. Factors influencing the outcome and duration of removable appliance treatment. Eur J Orthod. 1994; 16: 181-6.

21- Küçükkeleş N, Ilhan I, Orgun IA. Treatment efficiency in skeletal Class II patients treated with the jasper jumper. Angle Orthod. 2007; 77:449-56.

22- Mavropoulos A, Karamouzos A, Kiliaridis S, Papadopoulos MA. Efficiency of noncompliance simultaneous first and second upper molar distalization: a three-dimensional tooth movement analysis. Angle Orthod. 2005; 75:532-9.

23- McKinney JR, Harris EF. Influence of patient age and sex on orthodontic treatment: evaluations of Begg lightwire, standard edgewise, and straightwire techniques. Am J Orthod Dentofacial Orthop. 2001; 120: 530-41.

24- McSherry PF, Bradley $\mathrm{H}$. Class II correction-reducing patient compliance: a review of the available techniques. J Orthod. 2000; 27:219-25.

25- Moullas AT, Palomo JM, Gass JR, Amberman BD, White J, Gustovich D. Nonsurgical treatment of a patient with a Class III malocclusion. Am J Orthod Dentofacial Orthop. 2006; 129: S111-8. 26- Nangia A, Darendeliler MA. Finishing occlusion in Class II or Class III molar relation: therapeutic Class II and III. Aust Orthod J. 2001; 17:89-94.

27- Pinskaya YB, Hsieh TJ, Roberts WE, Hartsfield JK. Comprehensive clinical evaluation as an outcome assessment for a graduate orthodontics program. Am J Orthod Dentofacial Orthop. 2004; 126: 533-43.

28- Pinzan-Vercelino CR, Janson G, Pinzan A, Almeida RR, Freitas MR, Freitas KM. Comparative efficiency of Class II malocclusion treatment with the pendulum appliance or two maxillary premolar extractions and edgewise appliances [corrected]. Eur J Orthod. 2009; 31:333-40.

29- Richmond S, Shaw WC, O'Brian KD, Buchanan IB, Jones R, Stephens CD, et al. The development of the PAR index (Peer Assessment Rating): reliability and validity. Eur J Orthod. 1992; 14: 125-39.

30- Skidmore KJ, Brook KJ, Thomson WM, Harding WJ. Factors influencing treatment time in orthodontic patients. Am J Orthod Dentofacial Orthop. 2006; 129:230-8.

31- Stalpers MJ, Booij JW, Bronkhorst EM, Kuijpers-Jagtman AM, Katsaros C. Extraction of maxillary first permanent molars in patients with Class II Division 1 malocclusion. Am J Orthod Dentofacial Orthop. 2007; 132:316-23. 